\title{
THEORETICAL RESEARCH ON EVOLUTION OF HEALTH OF PLANTS AFFECTED BY HEAVY METAL ABSORPTION PROCESS
}

\author{
Petru Cardei, Catalina Tudora \\ National Institute of Research-Development for Machines and Installations \\ Designed to Agriculture and Food Industry, Romania \\ petru_cardei@yahoo.com,cmc_tudora@yahoo.com
}

\begin{abstract}
The paper presents a modified version of the mathematical model of the heavy metal transfer process. The changes start from the beliefs of the authors that the factors that govern the plant's evolution (germination, growth and vegetative development, senescence and death) are environmental factors (structure and chemical composition of soil and air, their humidity, soil $\mathrm{pH}$, lighting, applied fertilization, meteorological events, etc.) and not the time. Time is an artificial parameter introduced to provide a simpler reporting of dynamic processes. We do not know if time has simplified, or if it kept the truth in reporting the results, but it is the most common parameter used for this purpose. Of the influential factors, only the temperature and the concentration of heavy metal ions in the soil are considered in this article. The paper presents simulations using minimal experimental data, model constants being obtained by calibration. Effects of increasing delay and triggering of the biomass descending process were achieved (phytoremediation also). The experiments required to increase the performance of the model, the theoretical and practical efficiency of such attempts are estimated. These appreciations allow readers to reflect, to try to estimate the theoretical and practical efficiency of such attempts.
\end{abstract}

Keywords: plants, bioaccumulation, disease, models, simulation.

\section{Introduction}

The paper presents a modified version of the mathematical model of the heavy metal transfer process developed in [1] and [2]. Mathematical models of heavy metal bioaccumulation are often simplified as much as possible to make them more intuitive, but also because the simple model requires less experimental data. The process of bioaccumulation of heavy metals in plants is a very complex one, which influences the physiological processes: feeding, development, quantity and quality of accumulated biomass, life span, etc. For these reasons, in this paper we have opted for a slightly more complex bioaccumulation model than, for example, in [3]. In turn, this model has been modified by introducing the influence factors that reflect the impact of the environmental factors to the plant life. This approach is in line with the authors' belief that time is not the parameter that makes the plant to evolve, but the effective control factors for the system: temperature, illumination, soil $\mathrm{pH}$ or development environment, environmental conductivity, rainfall regime, fertilization, etc. The environmental or management command factors can be expressed through a product with the role of introducing energy into the plant's bio-system, in various forms, energy harnessed by the plant by growth, generally by evolution (vegetative development). Besides these factors, there are other environmental factors that also provide energy elements that are likely to be unsuitable for the biosystem and which lead to nutritional deficiencies leading to slowing growth, various diseases, etc.

The mathematical modelling of bioaccumulation of heavy metals aims both to know the mechanisms of this process, but rather to identify ways of limiting or optimizing the bioaccumulation and bioremediation processes that plants have in soils.

The models presented in this article develop growth models with influence and bioaccumulation factors exposed in our latest papers, [3-6]. The models proposed in this paper, like the mathematical model from [4], are obtained from a basic model, in the form found in [7], initially created in [1].

The first model that has been built is the one that highlights the action of the thermal regime (one of the main factors influencing the growth and development of plants, [8-11]). For this, growth and mortality rates become functions products by multiplication with the factors that depend on temperature. Similarly, by increasing the number of multiplicative components, the model can be developed to simulate the action of light, fertilizers, various concentrations of gas in soil and air, $\mathrm{pH}$ in soil, etc. This construction was made for algae in [4]. In the research presented in this paper, besides the temperature factor, another factor that depends on the heavy metal concentration and which represents the reaction of the plant to the presence of heavy metal ions in the soil was introduced. The latter is the second mathematical model presented in the article. In the article [6] both stages of modelling are exposed separately. 
Starting from the model presented in this paper, by the same procedure as in [4], complex models can be obtained, in which the most important factors or even all the factors that influence the growth, vegetative development, senescence and plant death are shown. This addition will be made in the following articles. Building mathematical models focused on plant development in various environmental conditions and culture technologies has the advantage of compensating for the lack of experimental data by calibrating the model in relation to existing knowledge. The models thus obtained have the ability to be recalibrated, when new experimental data provide additional information.

\section{Materials and methods}

The mathematical model is presented in [7]. The lot of the parameters used in the mathematical model of heavy metal transfer in the soil-plant interaction is given in Table 1.

Table 1

\section{Parameters that are used in the mathematical model}

\begin{tabular}{|l|c|c|}
\hline \multicolumn{1}{|c|}{ Name } & Notation & Unit \\
\hline plant biomass & $T(t)$ & $\mathrm{kg} \cdot \mathrm{m}^{-2}$ \\
\hline concentration of metal ions in the plant & $S(t)$ & $\mathrm{mg} \cdot \mathrm{kg}^{-1}$ \\
\hline concentration of metal ions in the soil & $A(t)$ & $\mathrm{mg} \cdot l^{-1}$ \\
\hline concentration of hydrogen ions in the soil & $H(t)$ & $\mathrm{mg} \cdot{ }^{-1}$ \\
\hline time & $t$ & $\mathrm{years}$ \\
\hline flux of protons to the soil during rainfall & $W(t)$ & $\mathrm{mg} \cdot \mathrm{m}^{-2} \cdot \mathrm{year}^{-1}$ \\
\hline available water for roots & $p(t)$ & $\mathrm{mm}^{-1}$ \\
\hline coefficient of absorption & $\alpha$ & $1 \cdot \mathrm{kg}^{-1} \cdot \mathrm{year}^{-1}$ \\
\hline coefficient of leaching & $\beta$ & $\mathrm{year}^{-1}$ \\
\hline coefficient of reaction & $\phi$ & $\mathrm{year}^{-1}$ \\
\hline net growth function & $h(T)$ & $\mathrm{year}^{-1}$ \\
\hline $\begin{array}{l}\text { function of mortality or metabolic inefficiency of plants due to } \\
\text { the concentration of metal ions in plant }\end{array}$ & $\mu(S)$ & $\mathrm{year}{ }^{-1}$ \\
\hline Gaussian type function & $G$ & - \\
\hline Bump type function & $B$ & - \\
\hline temperature influence factors & $\Theta_{h}, \Theta_{\mu}, \Theta_{h S}$ & - \\
\hline $\begin{array}{l}\text { influencing factors giving the concentration of heavy metal in } \\
\text { the plant }\end{array}$ & $C_{h} C_{\mu}$ & - \\
\hline
\end{tabular}

In order to mathematically model the dynamic interaction between aluminium mobility, given by the soil acidity, and plants, [7] uses a mathematical model proposed in [1] and modified in [2], given by the following equation system:

$$
\begin{aligned}
& \frac{d T}{d t}=T(t)(h(T)-\mu(S)), \frac{d S}{d t}=\alpha A-h(T) S, \\
& \frac{d A}{d t}=\phi H-\beta A-\frac{\alpha A T}{p}, \frac{d H}{d t}=\frac{-\phi H}{9}-\beta H+\frac{W}{p}
\end{aligned}
$$

Because we did not have sufficient experimental information for calculating the coefficients for the growth and mortality rates given in [7], we used the hypothetical forms proposed in (4), for which the coefficients were calibrated. In other terms, the functions $h$ and $\mu$ are the growth and mortality rates of the plants.

The mathematical model (1), having modified growth and mortality rates according to (4), will be used to simulate the phenomenon of bioaccumulation of zinc in lettuce (Lactuca sativa L, fam. Asteraceae). Because the data required for the model are very large and there are no resources to obtain them (neither experimentally nor from the literature), general data on green salad culture will be used to calibrate the model. Some of the data will be used for calculating model constants, others for 
primary validation of the mathematical model. In addition, model (1) is made as a change that makes the biomass growth to be controlled by the temperature. This model is a first step towards introducing plant growth control functions: temperature, illumination, $\mathrm{pH}$, nutrients, etc. The presence of water is already introduced into the system, it remains to be seen later, if it can or may not be improved. In order to define the factors that express the influence of temperature in the mathematical model (1), we first introduce the Gaussian type function by a real variable, $y$ :

$$
G\left(p_{1}, p_{2}, p_{3}, p_{4}, y\right)=p_{1} \cdot \exp \left(\frac{-\left(y-p_{2}\right)^{2}}{2 p_{3}^{2}}\right)+p_{4},
$$

where $p_{i}, i=1, \ldots, 4$ are the parameters, and the bump function also of a real variable, $y$, is defined by the following formula:

$$
B\left(d, b_{1}, b_{2}, c_{1}, c_{2}, e_{1}, e_{2}, y\right)=\left\{\begin{array}{l}
G\left(d-e_{1}, b_{1}, c_{1}, e_{1} y\right), \text { if } y<b_{1} \\
d, \text { if } y \in\left[b_{1}, b_{2}\right] \\
G\left(d-e_{2}, b_{2}, c_{2}, e_{2} y\right), \text { if } y>b_{2}
\end{array},\right.
$$

where $d, b_{1}, b_{2}, c 1, c_{2}, e_{1}, e_{2}$, are the parameters that define favourable or unfavourable intervals for the process. For the study of the behaviour and of construction of these functions, you can consult [8].

For the growth and mortality rates, the following expression was used ( $U$ is a process parameter):

$$
h(U)=G\left(1, t_{0}, 0.5,0.001, U\right)+0.1, \mu(U)=9-G\left(1, t_{0}, 0.3,0.0000005, U\right) .
$$

The introduction of the temperature influence and of the metal concentration in the plant, in the mathematical model (1) is done by replacing the growth rate and the mortality rate given in (4) with their products with the factors that describe the influence of the temperature, given in (5), and the factors that describe the plant metal concentration, given in (6). The factors of thermal influence are defined by:

$$
\begin{aligned}
& \Theta_{h}(\theta)=B(1.5,15,25,1,1,0.5,0.5, \theta), \Theta_{\mu}(\theta)=4 \cdot\left(1.5-\Theta_{h}(\theta)\right) \\
& \Theta_{h S}(\theta)=B(0.005,15,55,10,10,0.001,0.0005, \theta), \theta \in[-30,60]
\end{aligned}
$$

and the influence factors of the metal concentration in the plant are defined by the formulas:

$$
C_{h}(\theta)=B(1,0,6,0.1,0.1,0.7,0.45, \theta), C_{\mu}(\theta)=B(0.006,0,6,0.1,0.1,0.3,0.3, \theta) .
$$

Under these conditions the model (1) is transform in the equations system (7):

$$
\begin{aligned}
& \frac{d T}{d t}=T(t)\left(h(T) \Theta_{h}(\theta(t)) C_{h}(S(t))-\mu(S) \Theta_{\mu}(\theta(t)) C_{\mu}(S(t))\right), \\
& \frac{d S}{d t}=\alpha A-h(T) \Theta_{h S}\left(\theta(t) C_{h}(S(t))\right) S, \\
& \frac{d A}{d t}=\phi H-\beta A-\frac{\alpha A T}{p}, \frac{d H}{d t}=\frac{-\phi H}{9}-\beta H+\frac{W}{p}
\end{aligned}
$$

\section{Results and discussion}

The model applies to the concrete data of the green lettuce crop, data taken from [12]. For the simulation, a lettuce culture was considered with a mean harvest mass of $0.45-0.9 \mathrm{~kg} \cdot \mathrm{m}^{-2}$, a density of 30000 to 80000 plants per hectare, the mass of 1000 seeds $0.9-1.2 \mathrm{~g}$ (900 seeds per $1 \mathrm{~g}$ ). It was considered to be an average of 8 plants per $\mathrm{m}^{2}$. Based on these data, the initial biomass value $T_{0}=9 \mathrm{mg} \cdot \mathrm{m}^{-2}$ was taken. The initial metal ion concentration in the plants is considered to be zero, $S_{0}=0 \mathrm{mg} \cdot \mathrm{l}^{-1}$, the initial metal ion concentration in the soil, $A_{0}=615 \mathrm{mg} \cdot \mathrm{l}^{-1}$, and the initial hydrogen ion concentration in soil, $H_{0}=0.001 \mathrm{mg} \cdot \mathrm{l}^{-1}$. The model constants (see Table 2) were chosen using the 
following information: duration of 50 to 60 days, the vegetation period (from sowing to the dismantling of the crop by harvesting the captains).

In the absence of concrete information, the system functions were delayed by the hypothesis: the proton flow in soil due to constant rain $\left(0.005 \mathrm{mg} \cdot\left(\mathrm{m}^{2} \cdot \text { year }\right)^{-1}\right)$, soil water available to roots with a sinusoidal distribution with a mean of $10 \mathrm{~mm}$ and the amplitude $7 \mathrm{~mm}$, the frequency 1,825 year $^{-1}$, and the growth and mortality rates, calculated after [2].

Table 2

\begin{tabular}{|c|c|c|c|}
\hline \multicolumn{4}{|c|}{ Characteristics of the lettuce crop (influenced/uninfluenced by metal bioaccumulation) } \\
\hline & years & months & days \\
\hline $\begin{array}{l}\text { Initial time (planting) measured from the } \\
\text { beginning of the year (January } 1 \mathrm{st} \text { ) }\end{array}$ & 0.246 & 2.957 & 89.998 \\
\hline End time (end of year) & 1 & 12 & 365.24 \\
\hline \multicolumn{4}{|c|}{ Reference data for the lettuce crop } \\
\hline Estimated harvest time & $0.426 / 0.533^{*}$ & $5.109 / 6.391$ & $155.51 / 149.51^{*}$ \\
\hline Production of lettuce harvested per $\mathrm{m}^{2}, \mathrm{~kg}$ & & 0.9 & \\
\hline Average mass of a lettuce plant, $\mathrm{kg}$ & & 0.113 & \\
\hline $\begin{array}{l}\text { Concentration of metal in the plant at } \\
\text { harvest, } \mathrm{mg} \cdot \mathrm{kg}^{-1}\end{array}$ & & $56.445 / 88.77$ & \\
\hline$\beta=0.44$ & $a=10$ & & $b=1$ \\
\hline
\end{tabular}

"The values placed after the slash line correspond to the salad crop affected by bioaccumulation of the metal.

The variation of the growth control function - the temperature, is given taking into account the average monthly temperature and the corresponding diurnal variation, according to the graphical representation in Figure 1. The soil $\mathrm{pH}$ variation was estimated from the concentration of hydrogen ions in the salts, resulting in the curve, also given in Figure 1. Figure 2 is shows the growing curve of the plant in two variants: witness variant (uncontaminated) and zinc-contaminated variant. It is noticed that the contaminated plant develops more slowly and reaches a maximum biomass value lower than the control variant (uncontaminated).

The simulation (solving the system of ordinary non-linear differential equations) for model (7) was done using the numerical methods of Mathcad 2001 software [13].
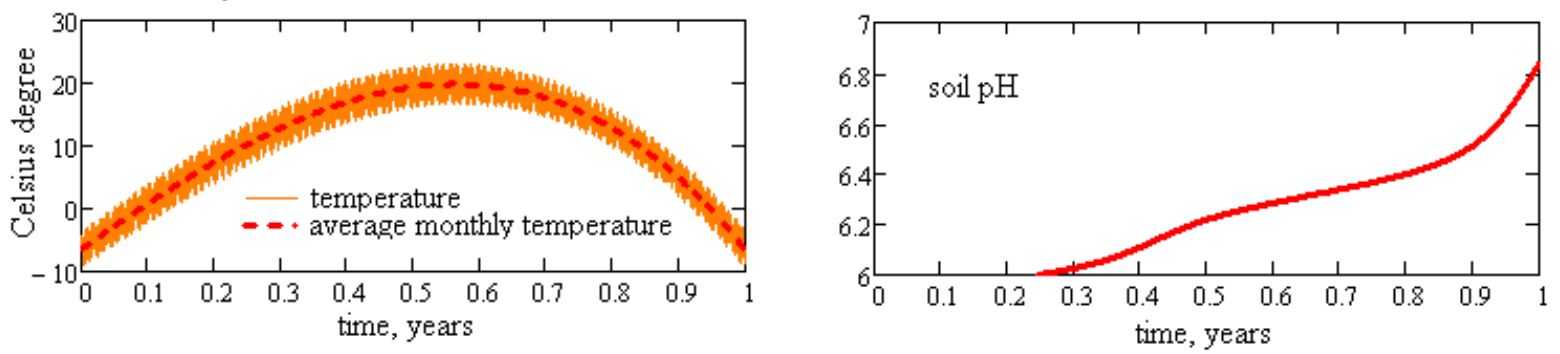

Fig. 1. Environment temperature (left), and soil pH (right), history

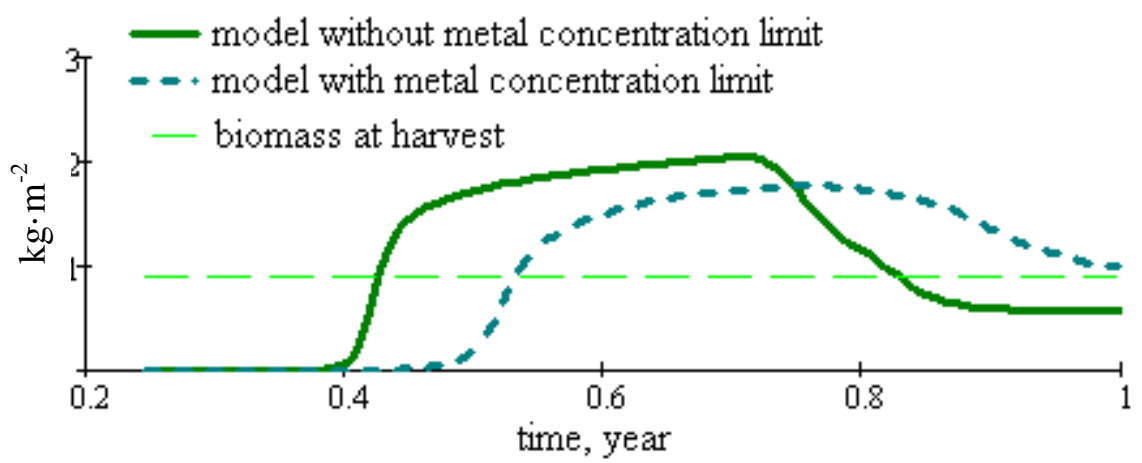

Fig. 2. Time dependence of biomass for uncontaminated and zinc-contaminated soil 


\section{Conclusions}

The model proposed by us succeeds in simulating some symptoms due to metal bioaccumulation: slowing of the vegetative growth process and the decrease of the biomass. Accumulation of heavy metals can be dangerous, when using plants as food or other purposes.

A possible application of the model is the optimization of the phytoremediation process. The model can predict the optimum harvesting (the stage, after which the bioaccumulation rate begins to decrease or parts of the plant die and can, in unwanted manner, reintegrate into the soil).

From the experimental point of view, for the improvement of such models, bioaccumulation experiments must be carried out for three to four generations of the plant. The effects of bioaccumulation in the offspring will be monitored. It is also necessary to determine the distribution of metal accumulation on the organs of the plant. The proposed model can be generalized for several factors that influence the plant growth and a wide range of metals. The ultimate desire is to express as many model parameters, depending on the quantified characteristics of "invariant events" and "intrinsic events" in the life of the biological entities that form the subject of the research. A development of this work is found in the preprint.

\section{Acknowledgements}

This work has been done within the project "Research regarding to development of mathematical models for assessing the impact of soil contamination on fruit and vegetables", contract 174/2017, within the PROGRAM 4, founded by UEFISCDI.

\section{References}

[1] De Leo G., Del Furia L., Gatto, M. The interaction between soil acidity and forest dynamics: a simple model exhibiting catastrophic behaviour. Theor. Popul. Biol. 43 (11), 1993, pp. 31-51.

[2] Guala S.D., Vega F.A., Covelo E.F. Modification of a soil-vegetation nonlinear interaction model with acid deposition for simplified experimental applicability. Ecol. Model. 220 (18), 2009, pp. 2137-2141.

[3] Sfiru R., Cardei P., Vladut V., Matache M., The role of mathematical modeling in research in the field of bioaccumulation of heavy metals, International Symposium ISB-INMA-THE, October 2017.

[4] Cardei P., Nedelcu A., A mathematical model for the evolution of Chlorella algae, preprint, January 2018. DOI: 10.13140/RG.2.2.29014.96320

[5] Nedelcu A., Cardei P., Ciuperca R., Researches on the cultivation of Chlorella vulgaris algae in a laboratory installation in aim to design a real-scale installation, preprint, January 2018, DOI: 10.13140/RG.2.2.35326.08009

[6] Cardei P., Tudora C., Simulation of mechanisms on the calculation and transfer of heavy metals in plants, 2018, DOI10.13140/RG.2.2.22779.82720

[7] Guala S. D., Vega F. A., Covelo E. F., The dynamics of heavy metals in plant-soil interaction, Ecological Modelling 221, Elsevier, 2010, p. 1148-1152.

[8] Hatfield J.L., Prueger J.H., Temperature extremes: Effect on plant growth and development, Weather and Climate Extremes Volume 10, Part A, 2015, pp. 4-10.

[9] Lafta A.M., Lorenzen J.H., Effect of High Temperature on Plant Growth and Carbohydrate Metabolism in Potato, Plant Physiology, 109, 1995, pp. 637-643.

[10] Wheeler T.R., Hadley P., Morison J.I.L., Elis R.H., Effects of temperature on the growth of lettuce (Lactuca sativa L.) and the implications for assessing the impacts of potential climate change, European Journal of AgronomyVolume 2, Issue 4, 1993, pp. 305-311

[11] Thompson H.C., Langhans R.W., Both A-J., Albright L.D.,Shoot and Root Temperature Effects on Lettuce Growth in a Floating Hydroponic Syatem, Journal of the American Society Horticultural Science 123(3), 1998, pp. 361-364.

[12] Cultura salatei in gradina si camp - tehnologie completa (Salad culture in the garden and field complete technology) [online][19.03.2018] Available at:

http://www.horticultorul.ro/legume/cultura-salatei-in-gradina_camp/ (In Romanian)

[13] MathSoft Engineering \& Education, Inc., Mathcad User's Guide with Reference Manual, Mathcad 2001 Professional, 2001. 